\title{
An illustration of the method of finite differences in the solution of Laplace's equation
}

\section{F J Mulligan}

\author{
Department of Experimental Physics, St Patrick's College, Maynooth, Co. Kildare, Ireland
}

Received 9 August 1991, in final form 30 September 1991

\begin{abstract}
A technique is described which illustrates the method of finite differences in the solution of Laplace's equation for a particular problem. The problem is to locate contours of equal electrical potential for a simple two-tube electrostatic lens. A short Bustc program which achieves this objective is provided together with sample results. The technique can be applied to many more complex problems.
\end{abstract}

\section{Introduction}

Almost every physics student comes into contact with Poisson's and Laplace's equations whether they be in electromagnetism, fluid dynamics or heat transfer. Various techniques for solving these equations are illustrated in a typical undergraduate course; in electromagnetism these might include the method of images, separation of variables and multipole expansions. These methods solve Laplace's or Poisson's equation analytically and they succeed only when the problem under consideration has certain degrees of symmetry. The only truly general method for solving static field problems is the method of finite differences, also known as the method of relaxation. This article is aimed at illustrating the method to today's undergraduates; it is particularly appropriate at present, since the method is most easily implemented on a computer-a tool which is available in almost all physics laboratories nowadays.

In the laboratory, the concept of contours of equal potential (equipotentials) and electric fields is frequently illustrated using some suitable analogue simulation, e.g. resistance (Teledeitos) paper (Ong 1983) or some form of electrolyte in a container to mimic the behaviour of the electric field. Karplus (1958) has provided an extensive review of many analogue simulation methods, including an error analysis of each method. This paper is concerned with using the method of finite differences to produce a set
Résumé. Description d'une technique qui demontre la méthode des differences finites dans la solution de l'equation Laplace pour un problème specifique. Le problème est de situer des contours d'un potential

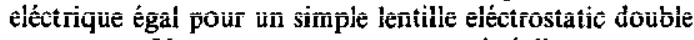
eprouvette. Un court program BASIC qui réalise cet objectif est compris, avec quelques examples. La technique peut-être employé en traitent des problèmes encore plus complexes.

of equipotentials with which the results from the

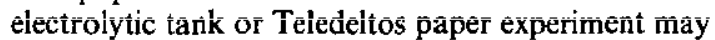
be compared. Descriptions of the electrolytic tank experiment can be found in most undergraduate texts (see e.g. Fewkes and Yarwood 1956). The measurements are easily made and results are readily obtained. In many laboratories, the physical situation is designed to match as closely as possible a problem which has an analytical solution. The measured potentials are then compared with the analytical solution in the discussion section of the experimental report. A different approach was adopted here in which it was decided to calculate the values of equipotential for the experimental situation in our laboratory using the method of finite differences. This is an intuitively satisfying way of calculating equipotentials, and it serves to introduce students to an exciting and useful method of solving problems. It also appears to give the students a better understanding of the equations governing the distribution of electric potential in space, and it is felt that the procedure represents a useful addition to the original experiment.

\section{Method of finite differences}

The method of solution using finite differences is well illustrated in Coulson and Boyd (1979), Grant and Phillips (1975) and Purcell (1965) to name a few, and only a brief explanation is given here. The problem 


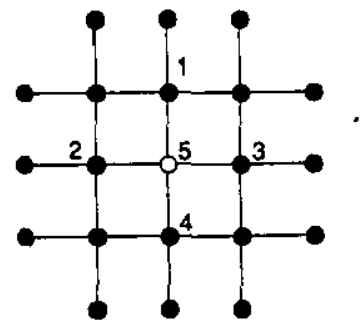

Flgure 1. An equally spaced mesh of points used in finding an approximate solution to Laplace's equation. The potential at point 5 is replaced by the average of the potentials at points $1-4$.

one wishes to solve is often a three-dimensional one with a degree of symmetry which allows it to be simplified to a two-dimensional problem. The Laplace or Poisson partial differential equation for the two remaining dimensions is then replaced by a finitedifference approximation in which the difference quotients are substituted for the actual derivatives. The problem is usually constructed on a lattice (or 'mesh') with points at regular intervals where the function of interest is to be evaluated. This represents a discretization of the problem. Numerous mesh types are available; three of the main ones-hexagonal, rectangular and triangular - are considered by Southwell (1946). Whether a square or triangular net is more suitable will depend upon the shape of the boundary. When the mesh is generated and the appropriate boundary conditions have been specified, the finite difference equations are set up for each mesh point and these are solved simultaneously to obtain a solution to the problem.

In rectangular coordinates, the finite difference form of Poisson's equation may be written as:

$$
V_{1}+V_{2}+V_{3}+V_{4}-4 V_{5}=h^{2}(\mathbf{R H})
$$

where the $V$ refer to the numbered points in figure 1 , $h$ is the vertical or horizontal separation of any two points, and RH is the value of the right-hand side of Poisson's equation at point 5 , when written in the form:

$$
\nabla^{2} V=\mathbf{R H}
$$

We have an equation like (1) above at each mesh point. These equations may be solved by an iterative method in the form:

$$
V_{s}^{n+1}=\frac{1}{4}\left[V_{1}^{n}+V_{2}^{n}+V_{3}^{n}+V_{4}^{n}-h^{2}(\mathbf{R H})\right]
$$

where $V_{1}^{n}$ is the $n$th iterate of the potential at $V_{1}$. The difference between the $n$th and $(n+1)$ th value, $\delta$, is calculated and this is compared with some predetermined minimum value; the iteration procedure is repeated across the whole array until the value of $\delta$ is less than the preset minimum value for all points, i.e. the iteration is said to have converged. Coulson and Boyd (1979) point out that convergence is guaranteed provided we move along grid lines, always in the same direction, as we calculate the potential at each new mesh point. Increased accuracy is obtained by reducing the mesh size $h$. If there are no sources of charge in the region we are considering, i.e. Laplace's equation, then $(\mathrm{RH})=0$ and the calculations required are reduced considerably.

\section{The experimental set-up}

Figure 2 shows a pair of coaxial conducting cylinders of equal diameter, $D$, separated by some distance which is usually specified in terms of $D$. This configuration of co-axial cylinders with a gap is important in electron optics as it is in fact an electrostatic lens (see e.g. Klemperer and Barnett 1971). The most frequently used arrangement has a gap of $0.1 D$. Many programs have been developed to perform matrix calculations to determine the path of electrons through such an arrangement. The maxtrix method is well illustrated in the treatise by Moore et al (1983). However, it is not the objective here to concentrate on the lens properties of the arrangement but rather to show how the lines of equipotential may be determined.

The situation depicted in figure 2 has cylindrical symmetry about the axis $a a^{\prime}$, and a knowledge of the distribution of potential along the length of the cylinders across any diameter is sufficient for a complete specification of the potential within the cylinders. The three-dimensional problem has thus been reduced to one having two dimensions. Our laboratory experiment simulates the two-dimensional

Figure 2. A typical two-cylinder electrostatic lens.

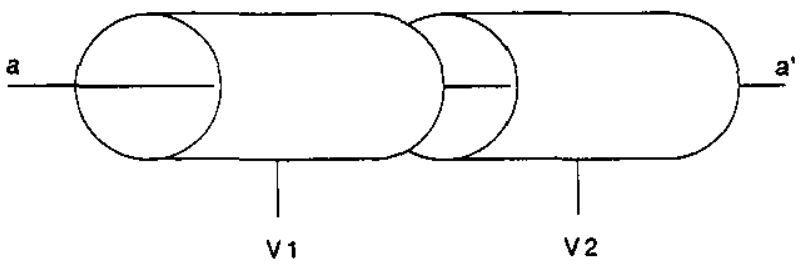


$\vee 1$

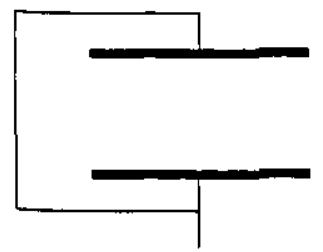

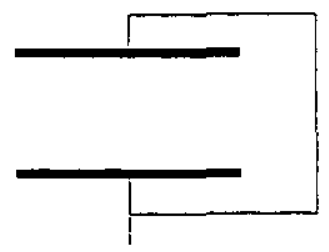

v2
Figure 3. The arrangement of electrodes in our electrolytic tank experiment.

geometry by having four planar electrodes held vertically in a shallow depth of electrolyte, and connected as shown in figure 3 . The shallow depth of electrolyte defines a plane and the parts of the left-handed pair of electrodes which are immersed in the electrolyte represent the two opposing sides of the left-hand cylinder along a diameter.

The problem must be set up by establishing the values of potential along the boundaries. The conductors and gap form the horizontal boundaries; the potentials at points in the gap are assigned by assuming a linear variation in potential between the edges of the conductors. The vertical boundaries of the problem are taken to be the ends of the cylinders away from the gap, and the potential at these ends is taken to be the potential of the cylinder itself. This is a very good approximation if the length of the cylinder is $3 D$ or more. In our case the lengths of the cylinders are only just over $1 D$, which does lead to some distortion of the outer equipotentials, but is only of the order of a few per cent.

\section{The sottware}

During the development of the program shown in figure 4, it was thought to be pedagogically useful to display the results after each individual pass of the relaxation algorithm. This is different from simply running a program and 'magically' getting a result at the end. A procedure called PRocshownUms was written which displays the two most significant digits of the value of the potential at each point. The decision to use this procedure constrains the number of points where the potential is calculated to 24 in the horizontal by 15 in the vertical. This rather small number limits the resolution which may be obtained from the method, but it was felt that demonstrating the principle was of greater importance than absolute accuracy in the result. The student can therefore observe the changes in the potentials as the relaxation algorithm proceeds. Figure $5(a)$ shows a print-out of the screen display immediately after the boundary values have been assigned. The potentials on the leftand right-hand electrodes were chosen as $0 \mathrm{~V}$ and $99 \mathrm{~V}$, respectively. Ideally, one would have used $0 \mathrm{~V}$ and $100 \mathrm{~V}$ for ease of interpretation of the matrix type print-out. Since PRoCshownums can only display the two most significant digits of the potential at each point, however, a value of $99 \mathrm{~V}$ was used for the right-hand cylinder as the best alternative. Figure $s(b)$ shows a dump of the screen display after the first pass of the relaxation algorithm, while figure $5(c)$ shows a similar plot after 70 iterations. The gap between the electrodes was taken as $0.1 D$ for the results shown.

On each iteration across the full set of mesh points, the program counts the number of points which have changed by more than some predetermined value of potential, DELTA. The value of DELTA is automatically assigned to be $0.01 \%$ of the difference in potential between the two cylinders (line 375 in the listing), as this was found to give good results. If DELTA is made smaller, the program will take longer to execute, but without any significant difference in the equipotentials. The iteration procedure may proceed stepwise, i.e. with $V_{x-1}^{n+1}$ being used in the calculation of $V_{x}^{n+1}$, or alternatively, each $V_{x}^{n+1}$ being calculated from its surrounding points from the $n$th iteration. The former approach has been adopted here. At the end of each iteration, the program displays the iteration number and the number of changes in that iteration. This procedure continues until an iteration is reached during which no changes have occurred. Figure $5(d)$ shows a print-out of the screen display after the iteration has converged.

The final step is to locate the points of equal potential from the points of known potential, and to then connect these in the form of contours. For each value of potential, $V$, the $x$ coordinate corresponding to each $y$ coordinate is determined by linearly interpolating between the two $x$ points whose potentials span the equipotential in question. When all the $x$ and $y$ coordinates for a given equipotential have been calculated, the points are simply joined by straight lines. The final plot may be dumped to a printer, and scaled to match the results of the experimental measurements. Figure 6 shows the result obtained for a pair of cylinders $10 \mathrm{~cm}$ in diameter with $a 1 \mathrm{~cm}$ gap and lengths of $11 \mathrm{~cm}$. The reason for such short lengths is due to the limitation imposed by the procedure PROCSHOWNUMS described above. In this figure, contours of equal potential are plotted for values of potential from $10 \%$ to $90 \%$ of the difference in potential between V1 and V2. This would correspond with a typical set of experimental results in the laboratory.

There are a number of improvements which could be made to the program as it is at present. The calculations could be speeded up by using the mid-plane symmetry of the experimental arrangement. This has not been implemented here as the program only takes a few minutes to complete, and over the period of a three hour practical, it is only a small fraction of the time. Using the mid-plane symmetry has an additional advantage in that it would allow the cylinder lengths to be longer, thus making the approximation, that the cylinder ends away from the gap have the potential of 
$10 \operatorname{DIM} P(25,15), \mathrm{RX}(15), \mathrm{RX}(15)$

20 REM PROG TO PLOT EQUIPOTENTIALS BY RELAXATION METHOD

50 MODE 0: PRINTTAB $(0,10)$ " "

60 INPUT "Enter Y-separation of plates in $\mathrm{cm}$. (MAX-15) ", YSPACE

100 INPUT "Enter potential on left hand cylinder ",vl

110 INPUT " Enter potential on right hand cylinder ",V2

120 INPUT "Enter the $X$-separation of the plates in cm. (MAX-13) "XSPACE

125 XSIZE $-22+(X S P A C E$ MOD 2 )

$130 X A-(X S I Z E-X S P A C E) / 2: X B-X A+X S P A C E$

150 CLS

160 REM-

170 REM SET ALL POTENTIALS $=0$

180 REM

190 FOR $X=0$ TO XSIZE:FOR $Y=0$ TO YSPACE: $P(X, Y)=0: N E X T$ Y:NEXT $X$

200 REM- REM SOUNDARY CONDITIONS

220 REM

230 FOR $X=0$ TO $X A$

$240 \quad P(X, 0)=V 1: P(X+X B, 0)-V 2$

$250 P(X, Y S P A C E)=V 1: P(X+X B, Y S P A C E)=V 2$

260 NEXT $X$

270 FOR $Y=0$ TO YSPACE

$280 P(O, Y)=V 1: P(X S I Z E, Y)=V 2$

290 NEXT $Y$

300 FOR $X=1$ TO XSPACE $: K=X+X A$

305 POTEN $=\mathrm{V} 1-(\mathrm{V} 1-\mathrm{V} 2) * \mathrm{X} / \mathrm{XSPACE}$

$310 P(R, 0)=$ POTEN

$320 P(K, Y S P A C E)=$ POTEN

330 NEXT $X$

340 PROCSHOWNUMS

350 REM-

360 REM RELAXATION ALGORITHM

370 REM

375 DELTA $=($ ABS (V1-V2) $) / 10000$

380 IT $=0: R E M$ IT is the iteration number

390 REPEAT

$395 \mathrm{CH}=0: \mathrm{REM} \mathrm{CH}$ is the number of changes in the current iteration

$400 \quad I T=I T+1$

410 FOR $X=1$ TO XSIZE- 1

420 FOR Y=I TO (YSPACE-1)

430 NEWVAL $=(P(X-1, Y)+P(X+1, Y)+P(X, Y-1)+P(X, Y+1)) / 4$

440 IF ABS (NEWVAL-P(X,Y)) < DELTA GOTO 470

$450 \mathrm{CH}-\mathrm{CH}+1$

$460 P(X, Y)=$ NEWVAL

470 NEXT $Y$

480 NEXT $X$

490 PROCSHOWNUMS

500 PRINT" "Iteration ";IT, CH, "changes"

510 UNTIL $\mathrm{CH}=0$

520 PRINT "ITERATION CONVERGED IN ";IT;" CYCLES"

525 WAIT $\$=$ INKEY\$ $(200)$

530 REM-

540 REM DRAW PLATES

541 XSCALE $=40$

542 YSCALE $=48.43$

550 CLS

560 REM

565 SPL-YSPACE*YSCALE

567 A $=0: B=3 * Y S C A L E: L=X A * X S C A L E: H=10$

570 PROCPLATE

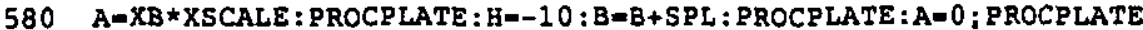

585 PRINT TAB $(0,29) ; " V 1=$ ";V1;" volts"; $\operatorname{TAB}(X B * 4,29) ; " V 2=$ ";V2;" volts"

590 REM-

600 REM PLOT EQUIPOTS

605 NPOTS $=10$

Figure 4. The computer program. 
610 VSTEP $=(V 2-V 1) / N P O T S$

$620 V 3=V 1+V S T E P: V 4=V 1+($ NPOTS -1$) * V S T E P$

630 FOR $V=V 3$ TO V4 STEP VSTEP

650 FOR $Y=1$ TO YSPACE 1

$660 \quad X=0$

665 REPEAT

670 DIFF $=A B S(V-P(X, Y)): N E W D I F F=A B S(V-P(X+1, Y))$

$680 x=x+1$

685 UNTIL NEWDIFF>DIFF

687 REM found nearest $x$ value

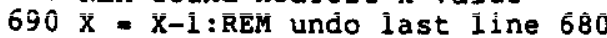

692 SIGN=1: VLTSTEP $=\operatorname{ABS}(P(X+1, X)-P(X, X))$

$694 \mathrm{IE}(((\mathrm{P}(\mathrm{X}+1, Y)<\mathrm{V})$ AND $(\mathrm{V}<\mathrm{P}(\mathrm{X}, \mathrm{Y})))$ OR $((\mathrm{P}(\mathrm{X}+1, \mathrm{Y})>\mathrm{V})$ AND $(\mathrm{V}>\mathrm{P}(\mathrm{X}, \mathrm{Y})))) \mathrm{GOTO} 700$

$696 \operatorname{SIGN}=-1: \operatorname{VLTSTEP}=\operatorname{ABS}(P(X-1, Y)-P(X, Y))$

$700 X 1=X+S I G N * D I F F / V L T S T E P: R X(Y)=X 1: R Y(Y)=Y$

710 PROCPOINT

740 NEXT $Y$

750 PROCCONNECT

760 NEXT V

770 END

771 REM

773 REM PROCEDURES DEFINED HERE

775 REM-

780 DEF RROCPLATE

790 MOVE $A, B: D R A W \quad A+L, B: D R A W$ A+L, B-H:DRAW A,B-H:DRAW A, B

800 ENDPROC

805 REM

810 DEF PROCPOINT

$820 I=X I * X S C A L E: J=(Y+3) * Y S C A L E$

830 MOVE (I-10), J : DRAW I+10,J:MOVE I, J-10:DRAWI, J+10

840 ENDPROC

845 REM---

850 DEF PROCSHOWNUMS

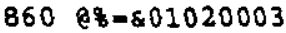

870 FOR $Y=0$ TO YSPACE

872 FOR $X=0$ TO XSTZE

874 PRINTTAB $(X * 3, Y \star 2) \quad P(X, Y) ;:$ NEXT X:PRINT:NEXT $Y$

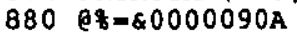

890 ENDPROC

895 REM

900 DEF PROCCONNECT

910 MOVE RX(1)*XSCALE, (RY (1)+3)*YSCALE

920 FOR $D=2$ TO YSPACE-1

930 DRAW RX(D)*XSCALE, (RY (D) +3)*YSCALE

940 NEXT D

950 ENDPROC

Figure 4. Continued

Figure 5. (a) Print-out of the screen display using the procedure procshownums immediately after the boundary potentials have been assigned. (b) As in (a) except that it shows the screen display after the first pass of the ielaxation algorithm across the two-dimensional array. (c) As in (b) except that 70 iterations have taken place on the array. (d) As in (b) except that the iteration process has converged after 108 passes.

(a)

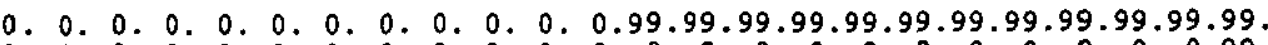

0.0 .0 .0 .0 .0 .0 .0 .0 .0 .0 .0 .0 .0 .0 .0 .0 .0 .0 .0 .0 .0 .0 .99$.

$0,0,0.0,0,0.0 .0 .0 .0 .0 .0 .0 .0 .0 .0 .0 .0 .0 .0 .0 .0 .0 .99$.

0.0 .0 .0 .0 .0 .0 .0 .0 .0 .0 .0 .0 .0 .0 .0 .0 .0 .0 .0 .0 .0 .0 .99$.

0.0 .0 .0 .0 .0 .0 .0 .0 .0 .0 .0 .0 .0 .0 .0 .0 .0 .0 .0 .0 .0 .0 .99$.

0.0 .0 .0 .0 .0 .0 .0 .0 .0 .0 .0 .0 .0 .0 .0 .0 .0 .0 .0 .0 .0 .0 .99 .

0.0 .0 .0 .0 .0 .0 .0 .0 .0 .0 .0 .0 .0 .0 .0 .0 .0 .0 .0 .0 .0 .0 .99 .

0.0 .0 .0 .0 .0 .0 .0 .0 .0 .0 .0 .0 .0 .0 .0 .0 .0 .0 .0 .0 .0 .0 .99 .

0.0 .0 .0 .0 .0 .0 .0 .0 .0 .0 .0 .0 .0 .0 .0 .0 .0 .0 .0 .0 .0 .0 .99 .

0.0 .0 .0 .0 .0 .0 .0 .0 .0 .0 .0 .0 .0 .0 .0 .0 .0 .0 .0 .0 .0 .0 .99$.

0.0 .0 .0 .0 .0 .0 .0 .0 .0 .0 .0 .99 .99 .99 .99 .99 .99 .99 .99 .99 .99 .99 .99 . 
(b)

0. 0. 0. 0. 0. 0. 0. 0. 0. 0. 0. 0.99.99.99.99.99.99.99.99.99.99.99.99.

$0.0 .0 .0 .0 .0 .0 .0 .0 .0 .0 .0 .25 .31 .32 .33 .33 .33 .33 .33 .33+33.58 .99$ :

0 . 0.0 .0 .0 .0 .0 .0 .0 .0 .0 .0 .6 .9 .10 .11 .11 .11 .11 .11 .11 .11 .42 .99 .

$0.0 .0 .0 .0 .0 .0 .0 .0 .0 .0 .0 .2 .3 .3 .4 .4 .4 .4 .4 .4,4.36 .99$.

0.0 .0 .0 .0 .0 .0 .0 .0 .0 .0 .0 .0 .1 .1 .1 .1 .1 .1 .1 .1 .1 .34 .99$.

0.0 .0 .0 .0 .0 .0 .0 .0 .0 .0 .0 .0 .0 .0 .0 .0 .0 .0 .0 .0 .0 .33 .99$.

0.0 .0 .0 .0 .0 .0 .0 .0 .0 .0 .0 .0 .0 .0 .0 .0 .0 .0 .0 .0 .0 .33 .99 .

0.0 .0 .0 .0 .0 .0 .0 .0 .0 .0 .0 .0 .0 .0 .0 .0 .0 .0 .0 .0 .0 .33 .99 .

0.0 .0 .0 .0 .0 .0 .0 .0 .0 .0 .0 .0 .0 .0 .0 .0 .0 .0 .0 .0 .0 .33 .99$.

0.0 .0 .0 .0 .0 .0 .0 .0 .0 .0 .0 .25 .31 .32 .33 .33 .33 .33 .33 .33 .33 .66 .99 .

0. 0. 0. 0. 0. 0. 0. 0.0.0.0.0.99.99.99.99.99.99.99.99.99.99.99.99.

Iteration 1

(c)

0. 0.0 .0 .0 .0 .0 .0 .0 . 0. 0. 0.99.99.99.99.99.99.99.99.99.99.99.99.

0. 0.1.1.1. 2. 3. 5. 7.10.17.31.67.81.88.91.94.95.96.97.98.98.99.99.

0 . 0. 1. 2. 3. 4. 6. 8.12.18.26.39.58.71.80.85.89.92.94.95.96.97.98.99.

0.1.1.2. 4. 6. 8.11.16.22.31.42.55.66.75.81.86.89.92.94.96.97.98.99.

0 . 1. 2. 3. 4. 6. 9.13.18.24.33.43.53.63.72.79.84.88.91.93.95.96.98.99.

0 . 1. 2.3. 5. 7.10.13.19.25.33.43.53.63.71.78.83.87.90.93.95.96.98.99.

0.1.2. 3. 4. 7.9.13.18.24.33.43.54.64.72.79.84.88.91.93.95.96.98.99.

0 . 1.2.3.4. 6.8.11.16.22.31.42.55.66.75.81.86.89.92.94.96.97.98.99.

0.1.1. 2. 3. 4. 6. 8.12.18.26.39.58.71.80.85.89.92.94.95.97.97.98.99.

0. 0. 1. 1. 1. 2. 3. 5. 7.10.17.31.67.81.88.92.94.95.96.97.98.98.99.99.

0 . 0. 0.0 .0 .0 .0 .0 .0 .0 .0 .0 .99 .99 .99 .99 .99 .99 .99 .99 .99 .99 .99 .99 .

Iteration $70 \quad 184$ changes

(d)

0. 0. 0. 0. 0. 0. 0. 0. 0. 0. 0. 0.99.99.99.99.99.99.99.99.99.99.99.99.

0. 0.1.1.2. 2. 3. 5. 7.11.17.31.68.82.88.92.94.95.96.97.98.98.99.99.

0.1 .1 .2 .3 .4 .6 .9 .13 .18 .27 .40 .59 .72 .80 .86 .90 .92 .94 .96 .97 .98 .98 .99 .

0. 1.2.3.4.6.9.12.17.23.32.43.56.67.76.82.87.90.92.94.96.97.98.99.

0.1.2.3. 5. 7.10.14.19.25.34.44.55.65.73.80.85.89.91.94.95.97.98.99.

0 . 1. 2. 4. 5. 7.10.14.20.26.35.44.54.64.72.79.84.88.91.93.95.97.98.99.

0.1.2.3.5.7.10.14.19.25.34.44.55.65.73.80.85.89.91.94.95.97.98.99.

$0.1,2,3,4,6,9=12,17,23,32,43,56,67,76,82,87,90,92,94.96,97,98.99$.

0.1.1. 2. 3. 4. 6.9.13.18.27.40.59.72.80.86.90.92.94.96.97.98.98.99.

0. 0.1.1.2. 2. 3. 5.7.11.17.31.68.82.88.92.94.95.96.97.98.98.99.99.

0.0 .0 .0 .0 .0 .0 .0 .0 .0 .0 .0 .99 .99 .99 .99 .99 .99 .99 .99 .99 .99 .99 .99 .

Iteration $108 \quad 0$ changes

ITERATION CONVERGED IN 108 CYCLES

Figure 5. Continued

the cylinder, more accurate. Additionally, all the points inside the boundary were set initially to zero for simplicity. Again this could be speeded up by an intelligent guess but is not a pre-requisite. Klemperer and Barnett (1971) provide a useful summary of various considerations in optimising the speed of convergence of the relaxation algorithm.

Very sophisticated programs exist to carry out the type of calculation described here, e.g., the Stanford Linear Accelerator Centre ray tracing program written by Hermannsfeldt (1979). It has as its first part a section to establish the boundary potentials followed by an interation using the method of finite differences. The input parameters for the Hermannsfeldt program is very much more complicated than the simple program given here and would not be suitable for a two session laboratory experiment.

\section{Conclusion}

It is felt that this addition to the standard experiment of plotting equipotentials is instructive in the study of methods of solving Laplace's and Poisson's equations. 


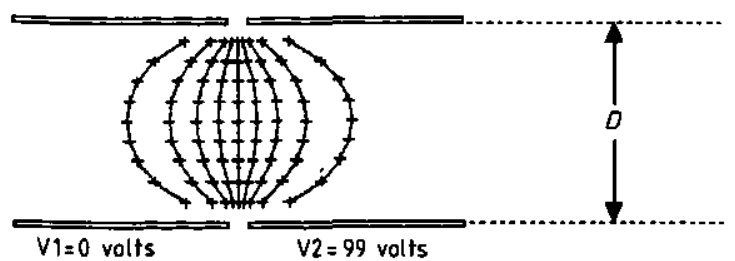

Figure 6. Print-out of the plotted contours of equipotential for our two-cylinder experimental arrangement.

It brings to light the sometimes rather dull subject of equipotentials and illustrates the method of finite differences, using the procedure PROCSHOWNUMS. It introduces the concepts of iteration and speed of convergence to a solution, and it is found to be an intuitively satisfying method of solving a complex problem. The method described can be readily adapted to almost any computer system, a listing of the programme which produced figures 5 and 6 using a BBC microcomputer is given in figure 4 . The comment lines may need to be removed if available memory is limited.

\section{Acknowledgement}

The author thanks D B Doyle for the preparation of a number of diagrams and for useful discussion of results.

\section{References}

Coulson D A and Boyd T J M 1979 Electricity (London: Longmans)

Fewkes J H and Yarwood J 1956 Electricity and Magnetism (London: University Tutorial Press)

Grant I S and Phillips W R 1975 Electromagnetism (London: Wiley)

Hermannsfeldt W B 1979 Report No. 226 Stanford Linear Accelerator Centre

Karplus W J 1958 Analogue Simulation (New York: McGraw-Hill)

Klemperer O and Barnett M E 1971 Electron Optics (Cambridge: Cambridge University Press)

Moore J H, Davis C C and Coplan M A 1983 Building Scientific Apparatus (London: Addison Wesley)

Ong P P 1983 Phys. Educ. 18 272-5 and references therein Purcell E M 1965 Electricity and Magnetism (New York: McGraw-Hill)

Southwell R V 1946 Relaxation Methods in Theoretical Physics (Oxford: Oxford University Press) 